\title{
Las TIC como elemento de cohesión en el desarrollo de la oralidad mediante el ABP. Un estudio de caso.
}

\author{
Manuel Francisco Romero Oliva \\ Universidad de Cádiz. Departamento de Didáctica de la Lengua y la Literatura \\ manuelfrancisco.romero@uca.es \\ Hugo Heredia Ponce \\ Universidad de Cádiz. Departamento de Didáctica de la Lengua y la Literatura \\ hugo.heredia@uca.es \\ Ángel Ordóñez Cruz \\ Universidad de Cádiz \\ angelordcruz@gamail.com
}

Fecha presentación: 01/11/2017 | Aceptación: 15/11/2017 |Publicación: 21/12/2017

\begin{abstract}
Resumen
Este estudio recoge las evidencias obtenidas de un estudio de caso realizado en un curso de $5^{\circ}$ de Educación Primaria donde se analizaron las aportaciones de las TIC en el desarrollo de la competencia comunicativa oral del alumnado desde una metodología integradora como es el ABP. El diseño de diversas estrategias para la recogida y análisis de datos -rúbricas, entrevistas no estructuradas o listas de observación- puso de manifiesto que las TIC contribuyeron a mejoras significativas de su propio desempeño -al "saber", al "saber hacer" y al "saber ser"-, especialmente en las dimensiones comunicativa y digital.
\end{abstract}

Palabras clave: TIC, ABP, currículo integrado, oralidad, investigación educativa

\section{Resum}

Aquest estudi recull les evidències obtingudes d'un estudi de cas realitzat en un curs de 5è d'Educació Primària on es van analitzar les aportacions de les TIC en el desenvolupament de la competència comunicativa oral de l'alumnat des d'una metodologia integradora com és I'ABP. El disseny de diverses estratègies per a la recollida i anàlisi de dades rúbriques, entrevistes no estructurades o llistes d'observació- va posar de manifest que les TIC van contribuir a millores significatives del seu propi acompliment -al "saber", al "saber fer" i al "saber ser "-, especialment en les dimensions comunicativa i digital.

Paraules clau: TIC, ABP, currículum integrat, oralitat, investigació educativa

\section{Abstract}

This study collects the evidence obtained from a case study carried out in a 5th grade Primary Education course where the contributions of ICT in the development of the oral communicative competence of the students were analyzed from an integrative methodology such as the PBL. The design of various strategies for the collection and analysis of data hubs, non-structured interviews or observation lists - showed that ICTs contributed to significant improvements in their own performance - to "knowing", "knowing" and "knowing" being "- especially in the communicative and digital dimensions.

Key Words: ICT, ABP, integrated curriculum, orality, educational research 
Romero Oliva, Manuel Francisco; Heredia Ponce, Hugo; Ordóñez Cruz, Ángel. "Las TIC como elemento de cohesión en el desarrollo de la oralidad mediante el ABP. Un estudio de caso”. @tic. revista d'innovació educativa. Número 19. Otoño (julio-diciembre 2017), pp. 30-39.

\section{Introducción}

Las Tecnologías de la Información y la Comunicación (en adelante, TIC) siguen incorporándose al quehacer escolar (Cabrero, 2004; Amar, 2006; Castells, 2006; Coll, 2009; Montolío, 2011; Cassany, 2012; Salzano, 2015). Estas herramientas, integradas a propuestas activas para el alumnado -como el Aprendizaje Basado en Problemas (en adelante, ABP)-, deben concebirse como una propuesta para el desarrollo de las competencias educativas (Torres, 1994; Perrenoud, 1997; Zabala y Arnau, 2007; Puig y Martín, 2007; Escamilla, 2009; Feito, 2010; Lomas, 2010; Majó, 2010).

Es, por ello, que el interés de este estudio reside en comprobar cómo pueden contribuir las TIC en la mejora de la competencia comunicativa oral a través de una propuesta de ABP -concretamente un Método de Proyectos de Kilpatrick (citado por Zabala y Arnau, 2014), con una duración estimada de 8 sesiones de 45 minutos en un aula de $5^{\circ}$ de Educación Primaria-, que contribuya a potenciar el espíritu crítico y social respecto a la violencia machista y la necesaria igualdad de derechos entre hombres y mujeres (Díaz, 2009; Real Decreto 126/2014).

\section{Marco Teórico}

Se El aprendizaje competencial se ha convertido en una exigencia en la escuela actual (Pasut, Ferreyra, Fontana, Perett y Ariel, 2008) pues contribuye a la formación del alumnado desde una triple dimensión -ser, saber y hacer-. En este sentido, la cohesión que ofrecen las TIC (Pérez Gómez, 2012; Marqués, 2013) en el desarrollo de metodologías activas, como ABP, y la enseñanza de lengua desde el enfoque comunicativo (Guerrero y Caro, 2015), se erigieron en un diseño adecuado para afrontar nuestro objetivo.

\subsection{La integración de las TIC}

Las tecnologías están vertebrando nuestra sociedad y requieren nuevas habilidades y destrezas para desarrollarnos y convivir dentro de ella, lo que demanda una alfabetización digital (Coll, 2009). En un futuro próximo, todas las personas deberán adaptarse a las herramientas tecnológicas para poder formar parte del grupo social (Mariño, 2009) y no ser víctimas de la exclusión que provoca la brecha digital (Cabero, 2004), sea cual fuere el origen de esta.

Estos flujos de creación y difusión de información nos acercan a la Sociedad del Conocimiento (Krüger, 2006; Amar, 2006) en la que todo es intercambio, interpretación y aplicación de información. En ella, las TIC ocupan un lugar en un universo social en continuo cambio determinado por la modernidad líquida (Bauman, 2006) que lo transforma a un ritmo tan trepidante que exige a las instituciones y a las personas estar actualizados para no quedar obsoletas.

Existe la urgente necesidad de adaptarse a los nuevos tiempos y demandas sociales, y la escuela debe acometer un proceso de reflexión e innovación sobre estas inercias. La vida se configura cada vez más en red (Castells, 2006), por lo que, si hay que educar para la vida, hay que educar para internet. Esa función socializadora de la escuela que ya apuntó Durkheim (1979) sigue vigente, lo que ha cambiado es la sociedad y sus reglas.

Sin embargo, este planteamiento no es sinónimo a pensar que las TIC serán una solución por sí mismas y que con su mera inclusión se solucionarán los problemas de cada centro educativo (Coll, 2009). Las tecnologías son un medio y no un fin, por lo que innovar es un proceso mucho más profundo en el que las tecnologías se convierten en una aliada para los cambios requeridos en todos los niveles (Mariño, 2009). Por tanto, no podemos olvidar que las TIC, como elementos de mejora en la escuela, solo refuerzan y promueven la innovación cuando se insertan en una dinámica de mejoras y cambio educativo más amplio (Cassany, 2012).

Y es que la tecnología, con su poder de seducción, ha llevado a generar ciertos mitos a su alrededor, algunos de los cuales han calado en el mundo académico. Uno de los más extendidos y peligrosos fue el de nativos digitales acuñado por Prensky (2001), que se ha ido desvirtuando con el tiempo. Es bien cierto que tanto los jóvenes como los niños actuales son usuarios de los nuevos medios tecnológicos de la información y la comunicación, pero ello no les hace intérpretes cualificados de sus significados (Salzano, 2015).

De esta manera, la denominación de nativos digitales puede llevarnos a pensar que adultos y escuela carecen de responsabilidad en lo que respecta a la educación en y para los nuevos medios, dado que los jóvenes nacen ya preparados para asumir los desafíos que suponen las TIC. Obviamente, no es lo mismo saber acceder a internet 0 manejar un smartphone que saber desenvolverse en los usos académicos de las TIC o contar con capacidad para aprender autónomamente con ellas (Cassany, 2012). De este modo, nos decantamos por denominarlos nativos digitales por expertos rutinarios (De la Torre, 2009).

Pese a todo, los avances producidos en las TIC permiten que el alumnado adquiera un papel más activo y formativo en el uso de la red. Así, los usuarios han pasado de ser consumidores del conocimiento, generado por otros; a ser productores de conocimiento y vivencias que comparten -la llamada Web 2.0 (O'Really, 2005)-: generando una cultura participativa similar a la estructura de una colmena de abeja (Cassany, 2012), dando lugar a un nuevo perfil de usuario-productor en red que ha venido a denominarse prosumidor (García, Ramírez y Rodríguez, 2014).

Por tanto, la escuela debe esforzarse en orientar a su alumnado en la demandada alfabetización digital, entendida no solo como el aprendizaje del uso funcional de estas tecnologías, sino también como el conocimiento de los significados y de las prácticas asociadas a dicha utilización con el fin de hacer un uso adecuado de ellas (Coll, 2009, 125). Con ello, nos aproximaríamos al verdadero desarrollo de lo que en el ámbito escolar europeo se ha denominado competencia digital: entre otros, uso seguro y crítico de las TIC con diferentes fines, desarrollo de habilidades técnicas básicas o capacidad de comunicarse e intercambiar información (Escamilla, 2009).

Esta acción se hace necesaria en nuestros días ya que diversos estudios demuestran que las TIC no se han integrado plenamente en nuestras escuelas y que, en muchos, casos, cuando se ha hecho, no han venido sino a reforzar prácticas tradicionales medidas por un soporte digital (Coll, 2009). Por ejemplo, en Andalucía, su implantación ha sido muy heterogénea y bastante limitada en la mayoría de los casos (Méndez y Delgado, 2016). Y es que, pese a su valoración en alza como herramienta didáctica y a la paulatina dotación de recursos TIC en los centros, su utilización viene siendo 
Romero Oliva, Manuel Francisco; Heredia Ponce, Hugo; Ordóñez Cruz, Ángel. "Las TIC como elemento de cohesión en el desarrollo de la oralidad mediante el ABP. Un estudio de caso". @tic. revista d'innovació educativa. Número 19. Otoño (julio-diciembre 2017), pp. 30-39.

aún bastante escasa (Trigueros, Sánchez y Vera, 2012). Son variados los escollos que impiden que la escuela camine al mismo ritmo que la sociedad en cuanto a las TIC: escasa inversión pública para la dotación de recursos (Méndez y Delgado, 2016), falta de formación docente sobre las nuevas tecnologías (Trigueros, Sánchez y Vera, 2012), tecnofobia o miedo al papel de estas herramientas por parte de los docentes (Pavón, 2013) o necesidad de actualización pedagógica con los nuevos escenarios que establecen estas tecnologías (Marqués, 2013).

Esta situación requiere la necesaria implicación del docente -instado a una actualización pedagógica-, la reclamada innovación educativa. Los ejemplos y experiencias en las aulas son cada día más numerosos y los estudios e investigaciones arrojan cada vez más luz sobre cómo concretar los currículos en metodologías acordes a este nuevo escenario (Marqués, 2013). Y, desde esta visión, las TIC adoptan un rol clave para el aprendizaje en la escuela (Coll, 2009).

Como ejemplo de este nuevo tiempo, existen interesantes propuestas focalizadas en el desarrollo de la competencia digital, en propiciar su alfabetización digital y en prepararlos para la vida que les ha tocado vivir, la exigente Sociedad del Conocimiento (Alcalá, 2015; Martínez y Barranco, 2013; Coll, Mauri y Onrubia, 2008). Estas medidas toman como referencia investigaciones a través de internet, la familiarización del alumnado con los blogs y su rol prosumidor (Romero y Vela, 2013) o las experiencias de "podcasting" (Martín, 2014), algunas sustentadas en el canal Youtube (Fernández y Vera, 2010).

En general, la integración de las TIC en los proyectos de Educación Primaria reporta ventajas tales como la motivación, el aprendizaje significativo, el papel activo del alumnado o la potenciación de otras competencias del currículo. De este modo, se deja a un lado la memorización de contenidos para dar paso a un aprendizaje más profundo y sustentado en la comprensión, donde el papel del alumno es clave durante todo el proceso, desarrollando competencias que le permiten adaptarse a los cambios permanentes de nuestra sociedad (Amar, 2006).

Se trata, en definitiva, de una redefinición de las experiencias de enseñanza-aprendizaje, del proceder didáctico docente y de las vivencias del alumno. Para el necesario cambio en las escuelas respecto a las TIC, se requieren ordenadores y conexión a internet en las aulas, pero también una formación profunda como docentes profesionales adaptados a su tiempo capaces de incluir nuevos hábitos de trabajo, minuciosas planificaciones $u$ optimizar los recursos digitales (Cassany, 2008).

\subsection{La oralidad en el aula}

El lenguaje es una herramienta básica del comportamiento social, un instrumento que configura al ser humano: sus ideas, su inteligencia, su personalidad... por lo que es esencial para el desarrollo de cualquier faceta de su existencia (Mendoza, López y Martos, 1996). En este sentido, Wittgenstein (1978) asociaba los límites del lenguaje de cada persona con los límites de su propio pensamiento.

Por este motivo, se antoja esencial que la escuela contemple su enseñanza desde el uso funcional de la lengua, conocer e interaccionar con el ser humano y desarrollarse en sociedad. De un modo u otro, el leguaje y la comunicación están en todas las aulas y materias, por lo que todos los docentes deben enseñar a comunicarse y contribuir a la competencia en comunicación y lingüística, dado que la lengua es en sí misma un vehículo para el aprendizaje (Cassany, Luna y Sanz, 2008).

La escuela queda configurada en este contexto como un agente de "normalización lingüística" con una importante responsabilidad sobre la lengua, debiendo ofrecer al alumnado un modelo estándar para su desarrollo y comunicación social, diferenciando, entre otros aspectos: lengua oral y escrita, registros, contextos, usos, tipologías textuales, etc. (Mendoza, López y Martos, 1996).

Pero, a pesar de los estudios de las últimas décadas y las aportaciones incorporadas de la lingüística (Lomas, 2010), la tarea de enseñar lengua en las escuelas sigue sin ser sencilla (Mendoza, López y Martos, 1996), hecho agravado por una cultura docente todavía con sesgos tradicionales; el desequilibrio en el tiempo dedicado al aprendizaje de las diferentes esferas de la lengua, priorizando la ortografía y la morfología sobre la oralidad; o el diseño organizativo de las aulas (Cassany, Luna y Sanz, 2008).

Ante este panorama, es necesario (re)plantear la lengua en los centros educativos. Su enseñanza debe posibilitar acciones comunicativas en diferentes contextos (Lomas, 2010). Por esta razón, "la lengua debe concebirse como parte integrante de la vida en sociedad" (Jakobson, 1984, 15): el uso y la comunicación para el desarrollo social del sujeto.

Pese a esclarecer cuál es el enfoque más apropiado, el desafío sigue sin ser sencillo, dado que desde la escuela actual hay que afrontar nuevos retos propios de nuestro tiempo (Prado, 2004) como: la superación de la tradición lingüística para dar mayor relevancia al papel funcional de la lengua; la preparación multidisciplinar del docente para ofrecer una formación pragmática al alumnado; la adaptación al Marco Común Europeo; la puesta en práctica de experiencias creativas e innovadoras; o la apertura a nuevos géneros literarios y a las TIC, entre otros.

Para ello, la toma de conciencia y formación de los docentes, además del reflejo de su trabajo en los centros, son requerimientos esenciales. En este sentido, el Plan de Centro (Cassany, Luna y Sanz, 2008) o iniciativas como el Plan Lingüístico de Centro impulsado desde la Junta de Andalucía (Romero y Trigo, 2015) pueden ser herramientas de concreción y orientación de gran valía para el trabajo en la escuela encaminado a la mejora de la capacidad comunicativa de los escolares, y haciendo hincapié en su carácter "transversal, transmisor e instrumental" (Mendoza, López y Martos, 1996, 35), dado que no puede haber en la escuela aprendizaje sin comunicación.

Hablamos, pues, de centrar los esfuerzos en enseñar a hablar más que en enseñar el sistema de la lengua, es decir, ubicar al alumno en un contexto comunicativo y significativo (el aula) donde pueda hacer uso de la lengua (Morote, 2015). De este modo, se contribuye verdaderamente al desarrollo de la competencia comunicativa (Hymes, 1971; Lomas, 2010; Pérez y Zayas, 2012), de marcado carácter transversal, que implica intercambio comunicativo, un contexto social o la adquisición de habilidades propias.

$\mathrm{Si}$ nos acercamos al Marco Común Europeo de Referencia para las Lenguas (MCERL), este determina la 
Romero Oliva, Manuel Francisco; Heredia Ponce, Hugo; Ordóñez Cruz, Ángel. "Las TIC como elemento de cohesión en el desarrollo de la oralidad mediante el ABP. Un estudio de caso". @tic. revista d'innovació educativa. Número 19. Otoño (julio-diciembre 2017), pp. 30-39.

enseñanza y la evaluación de la competencia lingüística "en relación con su aplicación comunicativa para una educación europea de calidad, movilidad" (Guerrero, 2009:17). De este modo, una educación que apueste por el desarrollo de esta competencia exige poner en marcha: metodologías activas, basadas en el aprendizaje del error; la resolución de problemas mediante proyectos; el desarrollo de un aprendizaje heurístico, autónomo, interactivo y cooperativo; crear un ambiente de socialización; o aplicar nuevas guías de innovación docente (Guerrero, 2009).

Por tanto, la competencia comunicativa oral adquiere su mayor dimensión en el uso y esto es, dicho de otro modo, en la interacción desde la escucha y el habla, siendo la lengua utilizada por el ser humano con un carácter “esencial y primariamente oral. Así lo prueba el hecho de que se aprende a hablar antes que a escribir y la existencia de lenguas en las que no existe la escritura" (Cifo, 2015:116). Así, los docentes han de ser conscientes de que el desarrollo competencial desde una óptica comunicativa requiere la adquisición, por parte de los alumnos, de microhabilidades propias de la oralidad, tales como reconocer, seleccionar, interpretar, anticipar, inferir y retener, para la comprensión; o planificar el discurso, conducir el discurso, negociar el significado, producir el texto y los aspectos no verbales que influyen en la interacción, para la producción (Cassany, Luna y Sanz, 2008), lo que permitirá al alumnado participar en intercambios comunicativos adecuadamente: con "coherencia, gramaticalidad, adecuación, cohesión, claridad y precisión, concisión y elegancia y, finalmente, cortesía" (Cifo, 2015:122).

Dentro de esta necesaria transformación, se exige una coherencia entre los fines y los medios que empleemos, por lo que se antoja esencial incluir las TIC en este proceso de transformación, ya que ayudará a plantear dentro del aula "una metodología activa y lúdica, donde se dinamicen las cuatro destrezas comunicativas -leer, escuchar, hablar y escribir- planteando la conversación como base de la interacción en el aula" (Romero, Jiménez, De Gracia y Salvador, 2011:33).

\subsection{El aprendizaje basado en proyectos}

Para desarrollar una propuesta didáctica en el aula que persiga conseguir cierto aprendizaje del alumnado, lejos de la clásica concepción instructiva de enseñar a los niños como si fueran recipientes vacíos a rellenar (Feito, 2010), deberíamos plantearnos una cuestión: ¿Cómo aprenden los niños? Para dar respuesta a esta pregunta, existe una tradición científica desde el ámbito de la sicología que puede resultar de utilidad.

Una de las teorías de referencia en el ámbito del aprendizaje escolar es la sicogenética de Piaget, sustento del constructivismo, centrada en "explicar de qué manera se dan los procesos y mecanismos en el sujeto que le permiten construir y adquirir los conocimientos" (Chávez, 2003:16). Piaget describe el conocimiento como una actividad estructurante, construido por el propio sujeto a través de diferentes procesos que involucran el desarrollo, la maduración, la asimilación o la acomodación, entre otros, y siempre en interacción con su medio ambiente (Piaget, 1980).

Igualmente, relevante desde el punto de vista educativo fue la aportación de Vygotski (1979), que introduce el factor social en el aprendizaje y en la interacción con los demás para desarrollar en el individuo su conocimiento del mundo. Por tanto, la comunicación adquiere una dimensión esencial en el aprendizaje para alcanzar la Zona de Desarrollo Próximo.

Posteriormente, Bruner y Díaz (2000) toman el testigo de este enfoque centrado en el alumno como constructor de su propio conocimiento y aportan nuevas ideas, como la relevancia del descubrimiento, la formación de hipótesis por parte de los aprendices o la cultura que rodea al individuo como factor configurador de su mente. Estos autores incluyen también la acción, las imágenes y los símbolos como sistemas para representar la realidad (Torres, 1994).

En esta línea de investigación centrada en el niño, Ausubel hace incidencia en la importancia de conectar el aprendizaje de nuevas ideas con conocimientos previos, estableciendo una nueva red significativa (Ausubel, Novak, y Hanesian, 1983). Por este motivo, las experiencias propias de los individuos son fundamentales para el desarrollo de su conocimiento y para la evolución de la complejidad de estos a fin de comprender el mundo (García Díaz, 1998).

Otra aportación es la teoría de las Inteligencias Múltiples (Gardner, 1998) que pone de manifiesto las distintas formas existentes de procesar la información y de aprender de los individuos ya que insiste en la idea de que todos los niños tienen la capacidad de solucionar problemas o elaborar bienes valiosos, aunque cada uno lo haga desde una forma de razonamiento diferente (Gardner, 1998).

Finalmente, en referencia al cambio estructural que han propiciado las TIC en nuestra sociedad y en nuestra forma de aprender, hemos de mencionar la teoría conectivista propuesta, entre otros, por George Siemens (2004), y que ofrece una mirada a las habilidades de aprendizaje propias de la nueva era tecnológica y que está estrechamente relacionada con la competencia digital.

Gracias a todas estas aportaciones, el concepto de enseñanza-aprendizaje ha cambiado $y$, por ende, las metas esenciales perseguidas desde las escuelas. Como ya hemos mencionado, en el actual marco europeo, las instituciones educativas proclaman el desarrollo competencial del alumnado, entendido como "aquello que necesita saber cualquier persona para dar respuesta a los problemas a los que se enfrentará toda su vida" (Zabala y Arnau, 2007:13), evidenciando su matiz práctico y orientado a saber responder a situaciones nuevas desde conocimientos ya adquiridos (Feito, 2010). De este modo, en los colegios se propicia un viraje hacia el aprendizaje por competencias, dado que esto garantiza la transferibilidad de conocimientos para resolver problemas de un mundo en constante cambio (Bauman, 2006). Por este motivo, el aprendizaje debe impregnarse de significatividad y de funcionalidad (Zabala y Arnau, 2007).

Resulta evidente que los problemas en la vida no vienen aislados, segmentados ni organizados por disciplinas. Todo suceso social es un suceso complejo plagado de interrelaciones, por lo que el enfoque más apropiado para comprender y afrontar los problemas de nuestro tiempo es el enfoque sistémico (Capra, 1998). De hecho, los estudios sicológicos apuntan en este sentido, ya que a medida que se va ampliando el peso otorgado a la interacción social, sus significados, ambientes, dimensiones... más necesaria se hace la defensa de unos currículos integrados (Torres, 1994). De este modo, la escuela se encuentra ante el desafío de propiciar el desarrollo integral del alumnado y el aprendizaje que le 
Romero Oliva, Manuel Francisco; Heredia Ponce, Hugo; Ordóñez Cruz, Ángel. "Las TIC como elemento de cohesión en el desarrollo de la oralidad mediante el ABP. Un estudio de caso”. @tic. revista d'innovació educativa. Número 19. Otoño (julio-diciembre 2017), pp. 30-39.

permita la construcción autónoma de respuestas adaptadas (Perrenoud, 1997).

En este sentido, el ABP es una opción que ofrece un modelo en el que los estudiantes trabajan de manera activa, plantean, implementan y evalúan aprendizajes que tienen aplicación en el mundo real, más allá del aula (Martí, 2010).

El aprendizaje por proyectos se erige, pues, en una herramienta ideal para el desarrollo de competencias en el alumnado y para dar respuestas a los retos del siglo XXI, orientándose siempre a la acción (Martí, Heydrich, Rojas y Hernández, 2010), es decir, a las competencias. Estos proyectos tienen un carácter integrador que facilita la adaptación de la experiencia escolar a la realidad humana, marcada por su carácter holístico (Torres, 1994).

Pese a que este discurso pueda sonar a actual, realmente no es nada nuevo. Ya Dewey realizó ciertos aportes que terminaron de cristalizar con el trabajo de su discípulo Kilpatrick en 1921 (Orellana, 1989) y su experiencia basada en el denominado Método de Proyectos. Este pretendió asemejar el trabajo de su aula a una investigación básica "desde donde se tuviera la posibilidad de observar las habilidades, capacidades y competencias que desarrollan los estudiantes con este tipo de metodología" (Orellana, 1989:2).

Esta nueva forma de proceder supuso un cambio en la manera de enseñar y aprender, una crítica al aprendizaje verbal y la mecanización de saberes; la aplicación de una metodología respetuosa con el alumno, una apuesta por la reflexión y una nueva redefinición de los roles de docente y discentes (Puig y Martín, 2007) y un equilibrio entre la experiencia social del alumnado y su vida individual (Zabala y Arnau, 2014).

Pese a sus beneficios, el trabajo por proyectos integrados sigue siendo reclamado en la escuela actual (Zabala y Arnau, 2007). Sin embargo, se empiezan a ver brotes y paulatinamente van desarrollándose buenas prácticas en las aulas, ejemplos de innovación y de aprendizaje no reñidos con la diversión, implicación e intereses de los niños (Domínguez, 2017). Se caracterizan también por su carácter heterogéneo y difícilmente clasificable (Parejo y Pascual, 2014), siendo diseños flexibles capaces de adaptarse a cada contexto y situación concreta (Majó, 2010).

Desde un punto de vista metodológico, el trabajo por proyectos integrados permite dar una respuesta globalizada a las demandas educativas del alumnado; desarrollar competencias; ofrecer una formación polivalente capaz de adaptarse y dar respuestas a un mundo cambiante; incluir aspectos sociales, tecnológicos y científicos en la educación; u otorgar más libertad y autonomía al alumnado en su proceso de aprendizaje, entre otros muchos beneficios (Campos, 2002).

Por tanto, si el trabajo docente es serio y está bien sustentado (Torres, 1994), los proyectos integrados reúnen los requisitos ideales para el desarrollo competencial del alumnado (Tippelt y Lindemann, 2007), que pasa a ser uno de sus objetivos fundamentales (Majó, 2010), logrando "alumnos cada vez más y mejores capacitados para desarrollarse integralmente interviniendo eficazmente en los diferentes ámbitos de la vida" (Zabala y Arnau, 2007:12).

\section{Resultados \\ 3.1 Objetivos}

La presente actuación persigue una serie de objetivos que complementan nuestra principal intención: evaluar cómo el uso de las TIC, desde una metodología basada en proyectos -ABP-, contribuye al desarrollo de las habilidades orales en los estudiantes de Educación Primaria. En concreto:

$\mathrm{O}_{1}$ : Estudiar diferentes posibilidades de aplicación de las TIC en el aula.

$\mathrm{O}_{2}$ : Contrastar su utilización en sus dinámicas de aula para el desarrollo de la oralidad.

$\mathrm{O}_{3}$ : Analizar las creencias del profesorado acerca del trabajo por proyectos.

\subsection{Población y muestra}

Esta investigación se desarrolló en el CEIP Horizontes Educativos ${ }^{1}$ de Trebujena, Cádiz en un aula de $5^{\circ}$ curso de Educación Primaria. Se trata de un estudio de caso (Rodríguez, Gil y García, 1999; Vázquez y Angulo, 2003; McMillan y Schumacher, 2005) con una muestra de doble naturaleza: por un lado, 15 alumnos -entre los que hay ocho niñas de entre 10 y 13 años; y seis niños, de entre los 10 y 11 años-; y, por otro, tres docentes -la tutora, que también imparte a los alumnos las asignaturas de Matemáticas, Lengua y Literatura, Ciencias Naturales, Ciencias Sociales y Música; otra maestra, que imparte Inglés; y una tercera, de Educación Artística, Plástica y Visual.

\subsection{Técnicas e instrumentos para comprender el contexto}

Las principales técnicas e instrumentos que se utilizaron para recopilar la información y realizar un riguroso planteamiento metodológico fueron las siguientes:

- Lista de observación para el aula: con el objetivo de calibrar el uso y calidad de la expresión oral que realizan los alumnos en clase, tanto en las áreas lingüísticas, como en las no lingüísticas. Este instrumento fue confeccionado tomando como referencia las aportaciones de Romero y Trigo (2015) y Barragán (2005).

- Entrevistas no estructuradas: confidencial (McMillan y Schumacher, 2005), destinada a los docentes para conocer las concepciones y experiencias sobre la Didáctica de la Lengua y el papel de la comunicación en la escuela; su conocimiento y nivel de familiarización con los proyectos integrados; y su grado de alfabetización digital y la utilización que realizan de herramientas TIC en su práctica profesional.

- Rúbrica de destrezas orales: con el fin de medir el nivel de destreza de cada alumno relativa a la comunicación oral, tanto expresiva como receptiva. Para su confección se utilizaron como base los criterios de valoración de la comunicación oral expuestos en Prado (2014). Esta misma rúbrica se volvió a pasar en la fase analítica para contrastar los resultados obtenidos.

- Diario de campo: desarrollado con la finalidad
${ }^{1}$ Nombre ficticio del centro en el que se ha desarrollado la
investigación para preservar su identidad. 
Romero Oliva, Manuel Francisco; Heredia Ponce, Hugo; Ordóñez Cruz, Ángel. "Las TIC como elemento de cohesión en el desarrollo de la oralidad mediante el ABP. Un estudio de caso". @tic. revista d'innovació educativa. Número 19. Otoño (julio-diciembre 2017), pp. 30-39.

de registrar toda la información obtenida durante los períodos de observación participante dentro del aula, según las premisas facilitadas por Vázquez y Angulo (2003).

El análisis estadístico de los datos se realizó con el programa SPSS (Statistical Package for the Social Sciences) en su versión 20.0.0 ha sido utilizado para el análisis estadístico de los datos del profesorado, lo que ha permitido contar con datos que sirvieron para contrastar con las creencias docentes y realizar un análisis más exhaustivo de la situación.

\subsubsection{Adentrándonos en el contexto}

Los momentos claves de la indagación se inician justo antes de comenzar con el proyecto integrado diseñado para determinar la influencia de las TIC en el desarrollo de la competencia comunicativa, específicamente en los relativos a las destrezas orales. Así, se tomó como referencia una primera rúbrica (Prados, 2014) -Figura 1que situó el problema de investigación. En concreto, se organizaron una serie de exposiciones orales grupales con intervenciones individuales de todos los alumnos- de trabajos de clase realizados previamente. En las mismas, el registro total de los 15 alumnos alcanzaba los 190 puntos (con un promedio de 12'6 puntos), alejado del nivel medio calibrado en dicha rúbrica para el desarrollo de desempeño -16 puntos-. De hecho, se corroboró que tan solo había dos alumnos que estaban por encima de la media, con 17 y 18 puntos respectivamente.

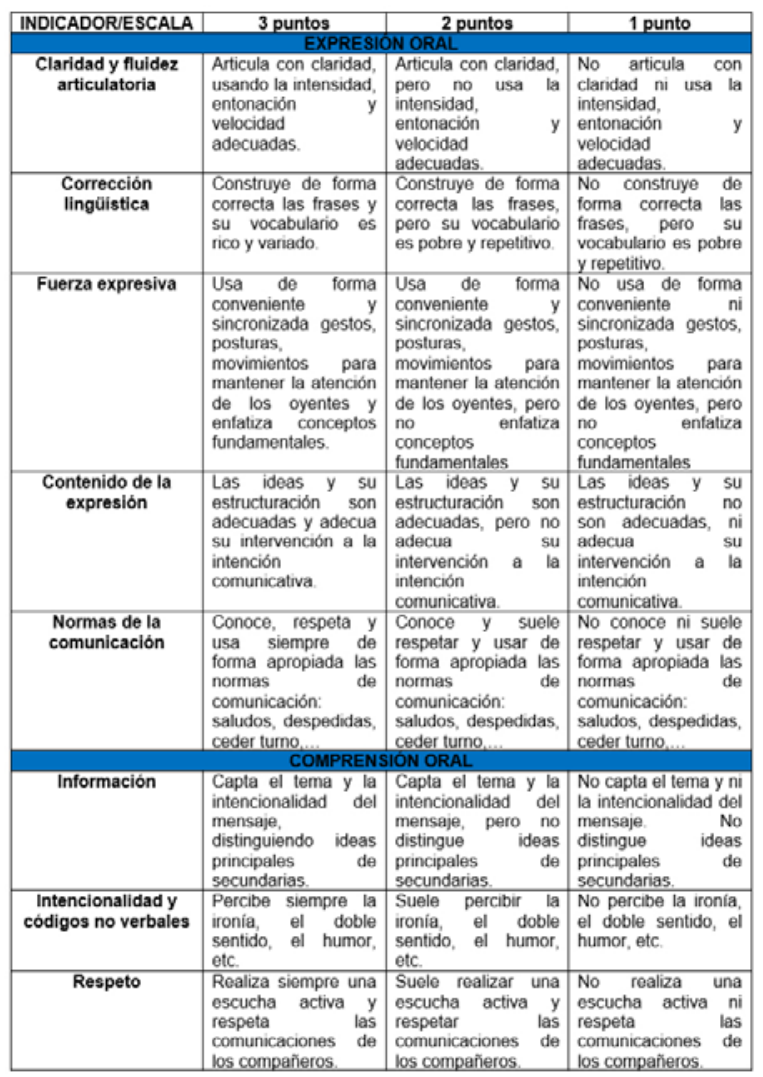

Figura 1. Rúbrica destrezas orales. Fuente: Adaptada Prado (2014)

Junto a ello, se diseñó una lista de observación -Figura 2que sirvió para indicar las prácticas letradas orales desarrollas por las docentes en las diferentes materias, corroborándose un índice poco relevante y centradas en aspectos académicos, incluida el área de Lengua Castellana.

\begin{tabular}{|c|c|c|c|c|c|c|c|}
\hline \multirow[b]{2}{*}{$\begin{array}{l}\text { HABLAR } \\
\text { PARA... }\end{array}$} & \multicolumn{2}{|c|}{ Área lingüustica } & \multicolumn{5}{|c|}{ Areas no linguisticas } \\
\hline & LENGUA & INGLÉS & MATEMATICAS & $\begin{array}{l}\text { CIENCIAS } \\
\text { SOCIALES }\end{array}$ & $\begin{array}{c}\text { CIENCIAS } \\
\text { NATURALES }\end{array}$ & PLÁstICA & MÚSICA \\
\hline $\begin{array}{l}\text { 1) Habbar para } \\
\text { regutar a viva } \\
\text { social escolar } \\
\text { yextreescolar }\end{array}$ & & & & & & & \\
\hline $\begin{array}{l}\text { 2) Hablar para } \\
\text { aprender y } \\
\text { aprender a } \\
\text { pensar }\end{array}$ & $x$ & $x$ & & $x$ & $x$ & $\mathrm{x}$ & \\
\hline $\begin{array}{l}\text { 3) Hablar para } \\
\text { leer y para } \\
\text { escribir pata }\end{array}$ & $x$ & $x$ & & & & & $x$ \\
\hline $\begin{array}{l}\text { 4) Hablar para } \\
\text { poder hablar } \\
\text { en pubblico }\end{array}$ & & & & & & & \\
\hline $\begin{array}{l}\text { 5) Hablar para } \\
\text { desarrollar } \\
\text { conocimientos }\end{array}$ & $x$ & & $x$ & $\mathrm{x}$ & $x$ & & $\mathrm{x}$ \\
\hline
\end{tabular}

Figura 2. Lista de observación de comunicación oral por materias. Fuente: Adaptada Romero y Trigo (2015) y Barragán (2005).

Estos datos aportaron ideas para nuestra toma de decisiones. De hecho, a partir de toda la información recabada, se elaboraron unas preguntas (entrevistas) destinadas a obtener nuevas evidencias y dirigidas a las maestras que impartían estas materias en la clase que ha sido objeto de estudio.

Este instrumento ofreció datos muy significativos y valiosas conclusiones para la investigación. Las docentes, con una experiencia que oscila entre los 6 y 9 años $(66,6 \%)$, conocían el trabajo por proyectos integrados solo por formaciones recibidas desde el Centro de Profesorado, y solo una lo había puesto en práctica, pese a que todas concebían esta metodología como "más atractiva, interesante y eficaz".

En cuanto a las TIC, las tres maestras las consideraban valiosas y se valían a menudo de ellas, aunque reconocieron que su Competencia Digital era "media" y que la mayoría (hasta el 66\%) solo la ponía en práctica en el aula a través de la pizarra digital.

Finalmente, en cuanto a la Competencia en Comunicación Lingüística, dos de las tres entrevistadas expresaron que la mayoría de sus alumnos tenía un nivel de desarrollo "medio", siendo curiosamente la maestra de Inglés (asignatura eminentemente comunicativa) la que considera ese nivel como "bajo". Respecto a la producción oral, el porcentaje se repite a pesar de que señalan que "intentan planificar e integrar el desarrollo de la expresión oral en todas las materias que imparten". Las otras dos compañeras reconocen que no la desarrollan de forma diseñada, sino que dejan que se genere naturalmente en el aula. En cuanto a su evaluación, el instrumento más utilizado por ellas es el registro de observación, aunque no es el único.

3.3.2 Diseñando desde la integración de estrategias

Ya en una segunda etapa, se aplicó la propuesta de intervención - 8 sesiones de 45 minutos-: se puso en práctica una metodología basada en proyectos integrados donde las TIC ocuparan un lugar destacado instrumentos como las tablets, el blog de aula, un canal Youtube, una cámara fotográfica para registrar los videos de los alumnos, la pizarra digital, etc-. Todo ello sin perder de vista el fin primordial de la intervención: intentar mejorar las destrezas de comunicación oral de nuestro alumnado -Figura 3-. 
Romero Oliva, Manuel Francisco; Heredia Ponce, Hugo; Ordóñez Cruz, Ángel. "Las TIC como elemento de cohesión en el desarrollo de la oralidad mediante el ABP. Un estudio de caso". @tic. revista d'innovació educativa. Número 19. Otoño (julio-diciembre 2017), pp. 30-39.

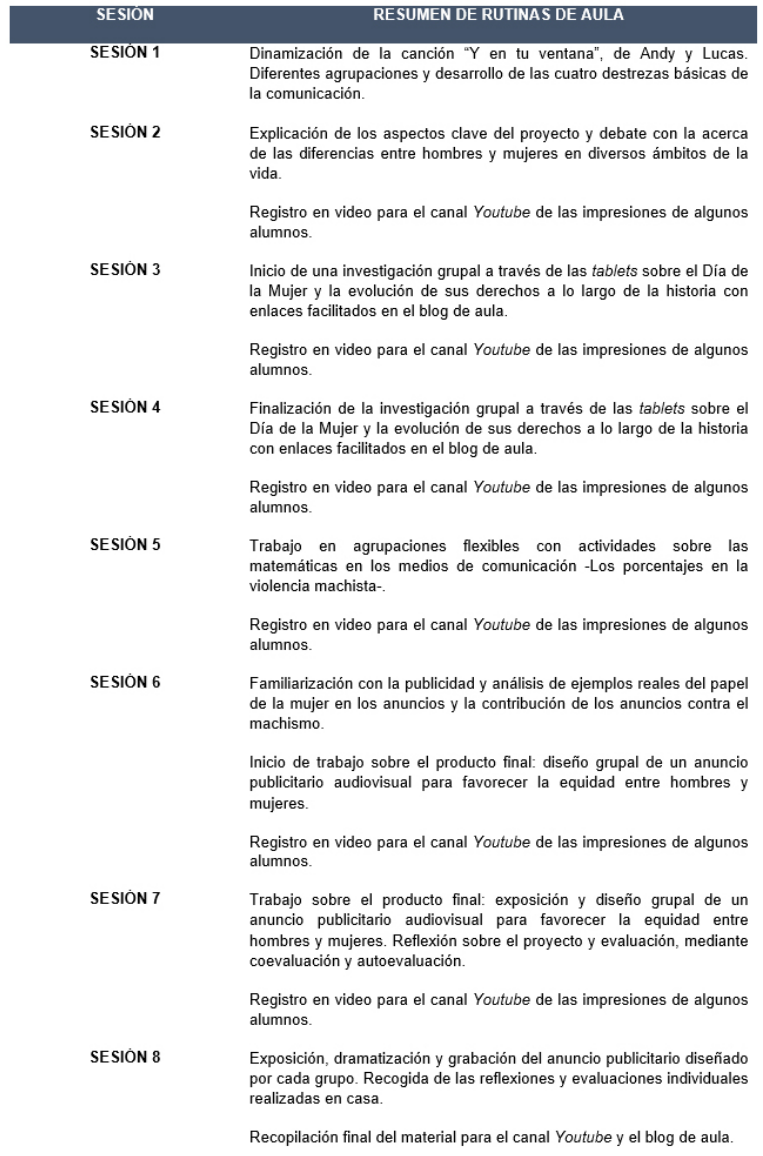

Figura 3. Cronograma de sesiones. Fuente: elaboración propia.

\section{Análisis y resultados}

Una vez finalizado el proyecto, se volvió a pasar al alumnado la misma rúbrica diseñada y ejecutada previamente, con la intención de evaluar el desarrollo de desempeño alcanzado. Los resultados fueron concluyentes: la suma total pasó de los 190 puntos iniciales a 246 puntos tras la experiencia desarrollada y la media se situó en 16, 4 puntos frente a los 12,6, siendo el porcentaje de desarrollo del 29,47\%. Esta progresión se produjo en todos los estudiantes -Figura 4-:

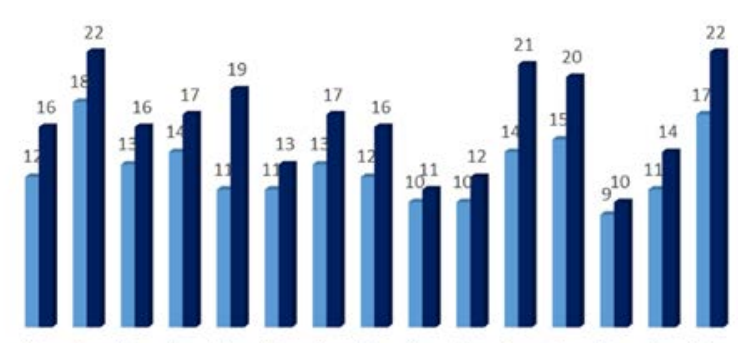

sissississors

Figura 4. Evolución individual de las destrezas orales. Fuente: elaboración propia.

Así las cosas, tras la propuesta de intervención en el aula, solo tres alumnos -9, 10, 13- evolucionaron muy poco (entre 1 y 2 puntos), aunque pese a todo progresaron; sin embrago, el resto desarrolló una progresión bastante aceptable; y cuatro estudiantes que tuvieron un crecimiento más que notable (de cinco o más puntos dentro de la rúbrica). El número de alumnos que igualaba o superaba el nivel medio de rendimiento se multiplicó por cinco -de dos pasaron a ser diez. A ello hay que sumar, además, algunos registros de destreza bastante desarrollada, habiendo ya niños que lograron alcanzar 20, 21 o 22 puntos sobre los 24 posibles de la rúbrica.

\section{Conclusiones}

La investigación desarrollada nos ha permitido obtener algunas conclusiones relativas a las incógnitas sobre las posibilidades de implementación de las TIC en Primaria, el desarrollo de las destrezas orales en la comunicación dentro del aula o el trabajo por proyectos.

Con este proyecto integrado, que ha contado como elemento transversal con la igualdad entre mujeres y hombres, se ha podido cumplir el objetivo principal de la indagación: el uso de las TIC desde una metodología basada en proyectos coadyuva con muy buenos resultados al desarrollo de las habilidades orales en los estudiantes de Educación Primaria.

La investigación ha permitido alcanzar los fines propuestos y los resultados de la propuesta han sido muy satisfactorios. Respecto a la metodología y la aplicación didáctica, se ha revelado que el trabajo por proyectos integrados y la implementación de las TIC son grandes potenciadores del aprendizaje competencial (en este caso comunicativo), ya que sitúan al alumnado en el centro de un proceso abierto y flexible, donde tienen autonomía y responsabilidad, y donde cuentan con posibilidades ilimitadas y oportunidades de reflejar su propio sentir gracias a las herramientas digitales.

Igualmente, se ha hecho evidente que las TIC se erigen en un instrumento que ayudan a motivar al alumnado y contribuyen a mejorar los procesos de enseñanzaaprendizaje al ofrecer una visión conectada y global de nuestro entorno, en sintonía con el enfoque basado en proyectos.

Las tareas propuestas han resultado bastante motivadoras para los estudiantes, aunque siempre existen alumnos más resistentes al cambio y acomodados en las dinámicas tradicionales de clase donde pueden asumir un rol más pasivo; sin embargo, incluirlos en la dinámica propuesta, escucharlos e individualizar la experiencia despertó su interés.

Por último, también se ha constatado la existencia de profesores que no abogan por evolucionar y mejorar en su práctica docente, ya que se encuentran demasiado estancados en el libro de texto como único recurso, el silencio como herramienta de control en el aula y la parcelación de conocimientos. En este caso, la apuesta por las TIC desde un enfoque integrado ha servido para el autocuestionamiento de dichas prácticas educativas y el comienzo de una indagación de nuevas formas de proceder en clase.

\section{Bibliografia}

Alcalá, D. (2015). "Análisis de la implicación y la regulación del trabajo del alumno mediante el uso de herramientas virtuales", Vivat Academia, 18, pp.82-112. DOI: 10.15178/va.2015.131.82-112

Amar, V.M. (2003). Comprender y disfrutar el cine. La gran pantalla como recurso educativo. Huelva: Grupo Comunicar. 
Romero Oliva, Manuel Francisco; Heredia Ponce, Hugo; Ordóñez Cruz, Ángel. "Las TIC como elemento de cohesión en el desarrollo de la oralidad mediante el ABP. Un estudio de caso". @tic. revista d'innovació educativa. Número 19. Otoño (julio-diciembre 2017), pp. 30-39.

Amar, V.M. (2006). "Las nuevas tecnologías y educación, sociedad y conocimiento", Comunicación y pedagogía: Nuevas tecnologías y recursos didácticos, 214, pp.14-18.

Amar, V.M. (2006). "Planteamientos críticos de las nuevas tecnologías aplicadas a la educación en la sociedad de la información y de la comunicación", Pixel-Bit. Revista de Medios y Educación, 27, pp79-87. https://goo.gl/xlsO67

Ausubel, D., Novak, J., y Hanesian, H. (1983). Psicología educativa: un punto de vista cognoscitivo. México: Trillas.

Barragán, C. y otros. (2005). Hablar en clase: Cómo trabajar la lengua oral en el centro escolar. Barcelona: Graó.

Bauman, Z. (2006). Modernidad líquida. Buenos Aires: FCE.

Bruner, J. y Díaz, F. (2000). La educación, puerta de la cultura. Madrid, Visor.

Cabero, J. (2004). "Reflexiones sobre la brecha digital y la educación" en Soto, F.J. y Rodríguez, J. (coords.), Tecnología, educación y diversidad: retos y realidades de la inclusión social. Murcia: Consejería de Educación y Cultura de Murcia, pp. 23-42. https://goo.gl/mgHL04

Campos, Y., (2002). "Ventajas del Currículum Integrado", Formadores Pedagogía, 1, pp.1-4. https://goo.gl/aGSnzc

Capra, F., (1998). La trama de la vida. Una nueva perspectiva de los sistemas vivos. Barcelona: Anagrama.

Cassany, D. (2012). En línea. Leer y escribir en la red. Barcelona: Anagrama.

Cassany, D., Luna, M., y Sanz, G. (2008). Enseñar lengua. Barcelona: Graó.

Castells, M. (2006). "Informacionalismo, redes y sociedad red: una propuesta teórica" en $\mathrm{M}$. Castells (Coord.), La sociedad red: una visión global. Madrid: Alianza, pp. 27-75.

Chávez, A. (2003). El método de proyectos: una opción metodológica de enseñanza en primer grado de Educación Primaria. Tesis doctoral, Universidad de Pedagogía Nacional, Culiacán, Sinaloa. https://goo.gl/jWOrAQ

Cifo, M. (2015). "Lengua Oral. Los procesos de comprensión y expresión orales", en P. Guerrero y M.T. Caro (Coords.), Didáctica de la lengua y educación literaria. Madrid, Pirámide.

Coll, C. (2009). "Aprender y enseñar con las TIC: expectativas, realidad y potencialidades", Boletín de la Institución Libre de Enseñanza, 72, pp.17-40. https://goo.gl/Z3oUlu

Coll, C., Mauri, M.T. y Onrubia, J. (2008). "Análisis de los usos reales de las TIC en contextos educativos formales: una aproximación socio-cultural", Revista electrónica de investigación educativa, 10, pp.1-18. https://goo.gl/pu4ZVz
De la Torre, A. (2009). "Nuevos perfiles en el alumnado: la creatividad en nativos digitales competentes y expertos rutinarios", Rusc, 6(1), pp.1-9. https://goo.gl/snrkoE

Díaz, M.J. (2009). "Prevenir la violencia de género desde la escuela", Revista de Estudios de Juventud, Universidad Complutense de Madrid, 86, pp.1-17. https://goo.gl/bVsU8F

Domínguez, N. (2017), "Investigar, expresarse y aprender a través de las ondas", Aularia, 1, pp.3540. https://goo.gl/NNUE93

Durkheim, E. (1979). La educación como socialización. Salamanca, Sígueme.

Escamilla, A. (2009). "Competencia en tratamiento de la información y digital. Concepto, componentes, relaciones y recursos", MULTlárea Revista de Didáctica, Escuela Universitaria de Magisterio de Ciudad Real, 4, pp.97-108. https://goo.gl/0XqGuh

Estado Español. Real Decreto 126/2014, de 28 de febrero, por el que se establece el currículo básico de la Educación Primaria. Boletín Oficial del Estado, 52, 19349-19420.

Feito, R., (2010). "De las competencias básicas al currículo integrado", Revista Qurriculum, 23, pp.55-79. https://goo.gl/eRhzGW

García Díaz, J. E. (1998). Hacia una teoría alternativa sobre los contenidos escolares. Sevilla: Diada Sevilla.

García, R., Ramírez, A. y Rodríguez, M.M. (2014). "Educación en alfabetización mediática para una nueva ciudadanía prosumidora", Comunicar, 43, pp.15-23. DOI: 10.3916/C43-2014-01

Gardner, H. (1998). Inteligencias múltiples: La teoría en la práctica. Barcelona: Paidós.

Guerrero, P. (2009). "La competencia comunicativa en la educación europea", MULTlárea Revista Didáctica, 4, pp.17-38.https://goo.gl/0XqGuh

Guerrero, P. y Caro, M. T. (2015). Didáctica de la lengua y educación literaria. Madrid: Pirámide.

Hymes, D. (1971). On communicative competence. Filadelfia, Universidad de Pensilvania.

Jakobson, R. (1984). "Poética", en Ensayos de lingüística general. Barcelona, Seix Barral.

Krüger, K. (2006). "El concepto de sociedad del conocimiento", Biblio 3W. Revista bibliográfica de geografía y ciencias sociales, XI, 683. http://www.ub.edu/geocrit/b3w-683.htm

Lomas, C. (2010). Cómo enseñar a hacer cosas con las palabras: Teoría y práctica de la educación lingüística. Barcelona: Paidós.

Majó, F. (2010). "Por los proyectos interdisciplinarios competenciales", Aula de Innovación Educativa, 195, pp.7-11. https://goo.gl/t9m8tR

Mariño, C. G. (2009). "TIC y la transformación de la práctica educativa en el contexto de las sociedades del conocimiento", DIM: Didáctica, 
Romero Oliva, Manuel Francisco; Heredia Ponce, Hugo; Ordóñez Cruz, Ángel. "Las TIC como elemento de cohesión en el desarrollo de la oralidad mediante el ABP. Un estudio de caso". @tic. revista d'innovació educativa. Número 19. Otoño (julio-diciembre 2017), pp. 30-39.

Innovación y Multimedia, 13, pp. 1-8. https://goo.gl/V9QtyT

Marqués, P. (2013). "Nuevas metodologías docentes para mejorar la formación y los resultados académicos de los estudiantes", Padres y Maestros, 351, pp.16-21.https://goo.gl/nL3zDB

Martí, J.A. (2010). Educación y Tecnologías. Cádiz: Universidad de Cádiz.

Martí, J.A., Heydrich, M., Rojas, M. y Hernández, A. (2010). "Aprendizaje basado en proyectos: una experiencia de innovación docente", Revista Universidad EAFIT, 46(158), pp. 11-21. https://goo.gl/hEhKdk

Martín, J. (2014). "Diseño de un proyecto de podcasting para la mejora de la expresión oral en la enseñanza de las lenguas", Revista DIM, 28, pp.1-15. https://goo.gl/N7r91T

Mcmillan, J. H., y Schumacher, S. (2006). Investigación educativa: Una introducción conceptual). Madrid: Pearson.

Méndez, J.M. y Delgado, M. (2016). "Las TIC en centros de Educación Primaria y Secundaria de Andalucía. Un estudio de casos a partir de buenas prácticas", Digital Education Review, 29, pp.134165. https://goo.gl/1Y9Bao

Mendoza, A., López, A. y Martos, E. (1996). Didáctica de la lengua para la enseñanza primaria $y$ secundaria. Madrid: Akal.

Montolío, M.C. (2011). Las TIC en la Educación Primaria en la provincial de Teruel. Tesis Doctoral, UNED, Facultad de Educación, Departamento de Didáctica, Organización Escolar y Didácticas Especiales. https://goo.gl/8UPr2u

Morote, E. (2015). "Fundamentación científica de la Didáctica de la Lengua" en P. Guerrero y M.T. Caro (Coords.), Didáctica de la lengua y educación literaria. Madrid: Pirámide, pp.24-44

O'Really, T. (2005). What is Web 2.0? [Mensaje en un blog].https://goo.gl/nWgyoD

Orellana, A. (1989). "El proyecto Kilpatrick, metodología para el desarrollo de competencias", Revista Clave XXI, Reflexiones y Experiencias en Educación, 1, pp.1-14. https://goo.gl/9mrkRT

Parejo, J.L., Pascual, C. (2014). "La Pedagogía por Proyectos: Clarificación Conceptual e Implicaciones Prácticas", CIMIE 14, Simposio, III Multidisciplinary International Conference on Educational Research, Segovia. https://goo.gl/vuDWmy

Pasut, M., Ferreyra, H. A., Fontana, M., Peretti, G. C. y Ariel, S. (2008). "De aprendizajes, competencias y capacidades en la educación primaria: Desandando caminos para construir nuevos senderos", Revista Iberoamericana de Educación, 47(3). http://rieoei.org/2702.htm

Pavón, F. (2013). "La introducción de las TIC en el curriculum y en la organización escolar de la Educación Infantil y Primaria" en Buenas prácticas educativas en el uso de las tic (1). Jaén: Joxman Editores, pp. 11-50.

Pérez Gómez, Á. I. (2012). Educarse en la era digital: La escuela educativa. Madrid: Morata.

Pérez, P. y Zayas, F. (2012). Competencia en comunicación lingüística. Madrid : Alianza.

Perrenoud, P. (1997). Construire des compétences dès l'école. París: ESF.

Piaget, J (1980). Seis estudios de psicología. Barcelona: Ariel.

Prado, J. (2004). Didáctica de la lengua y la literatura para educar en el siglo XXI. Madrid : La Muralla.

Prensky, M. (2001). "Digital natives, digital immigrants part 1", On the horizon, 9 (5), pp.1-6. DOI: $\underline{10.1108 / 10748120110424816}$

Puig, J.M. y Martín, X. (2007). Competencia en autonomía e iniciativa personal. Madrid: Alianza.

Rodríguez, G., Gil, J., y García, E. (1999). Metodología de la investigación cualitativa. Archidona: Aljibe.

Romero, M. y Trigo, E. (2015). "Herramientas para el éxito", Cuadernos de Pedagogía, 458, pp.1-5. https://goo.gl/KJ8Mk5

Romero, M., Jiménez, R., De Gracia, A., Salvador, A. (2011). La lectura expresiva en el aula. Propuestas de textos dialogados. Madrid: Visión Libros.

Romero, S., y Vela, M. (2013). "Edublogs musicales en el tercer ciclo de educación primaria: perspectiva de alumnos y profesores", Revista Complutense de Educación, 25, pp.195-221. https://goo.gl/kjpaiz

Salzano, D. (2015). "Repensar la educación en la era de los medios sociales", Hachetetepé: Revista Científica de Educación y Comunicación, 11, pp. 79-90. https://goo.gl/HECju6

Siemens, G. (2004). "Connectivism: A Learning Theory for the Digital Age" en Elearnspace, everything elearning. http://www.elearnspace.org/Articles/connectivism.h $\underline{\mathrm{tm}}$

Tippelt, R. y Lindemann, H. (2007). "El método de proyectos", APREMAT, 1, pp.1-14. https://goo.gl//SF9Wp

Torres, J. (1994). Globalización e interdisciplinariedad: el currículum integrado. Madrid: Morata.

Trigueros, F.J., Sánchez, R., y Vera, M.I. (2012). "El profesorado de Educación Primaria ante las TIC: realidad y retos" en Revista Electrónica Interuniversitaria de Formación del Profesorado, 15(1), pp.101-112. https://goo.gl/HyxwoM

Vázquez, R., y Angulo, F. (2003).Introducción a los estudios de casos: Los primeros contactos con la investigación etnográfica. Málaga: Aljibe.

Vygotski, L. S. (1979). El desarrollo de los procesos psíquicos superiores. Barcelona: Crítica. 
Romero Oliva, Manuel Francisco; Heredia Ponce, Hugo; Ordóñez Cruz, Ángel. "Las TIC como elemento de cohesión en el desarrollo de la oralidad mediante el ABP. Un estudio de caso". @tic. revista d'innovació educativa. Número 19. Otoño (julio-diciembre 2017), pp. 30-39.

Wittgenstein, L. (1978). Philosophical Investigations. Oxford: Blackwell.

Zabala, A. y Arnau, L. (2007). 11 ideas clave, cómo aprender y enseñar competencias. Barcelona: Graó.

Zabala, A. y Arnau, L. (2014). Métodos para la enseñanza de las competencias. Barcelona: Graó.

| Cita recomendada de este artículo

Romero Oliva, Manuel Francisco; Heredia Ponce, Hugo; Ordóñez Cruz, Ángel. (2017). "Las TIC como elemento de cohesión en el desarrollo de la oralidad mediante el ABP. Un estudio de caso”. en @tic. revista d'innovació educativa. Número 19. Otoño (Julio-Diciembre 2017), pp. X-X. 